\title{
Implementation of Model Predictive Control with Modified Minimal Model on Low-Power RISC Microcontrollers
}

\author{
Binh P. Nguyen, Yvonne Ho, Zimei Wu and Chee-Kong Chui \\ Department of Mechanical Engineering, National University of Singapore \\ 9 Engineering Drive 1, Singapore 117576 \\ \{phubinh, yvonne.yw.ho, g0800347,mpecck\}@nus.edu.sg
}

\begin{abstract}
Due to the ability of modeling multivariable systems and handling constraints in the control framework, model predictive control (MPC) has received a lot of interest from both academic and industrial communities. Although it is an established control technique, implementing MPC on small-scale devices is a challenge since we need to handle complicated issues of the control framework using limited computational power and hardware resources. This paper presents our implementation of MPC with constraints on the Texas Instruments MSP430 16-bit microcontroller platform. The MPC operational constraints which are supported in our design include rate of change, amplitude and output constraints, while the associated optimization problem is solved using a primal-dual interior-point algorithm based on predicator-corrector method. Our implementation is demonstrated in a prototype of a real-time closeloop blood glucose regulation system using a modification of the minimal model. Experimental results show that our system is able to achieve desired diabetes management, and the chosen microprocessor is capable of performing the MPC algorithm accurately with high energy-efficiency and in realtime.
\end{abstract}

\section{Categories and Subject Descriptors}

C.3 [Special-Purpose and Application-Based Systems]: Process control systems

\section{General Terms}

Design

\section{Keywords}

Model predictive control, glucose regulation, minimal model, embedded system.

\section{INTRODUCTION}

Permission to make digital or hard copies of all or part of this work for personal or classroom use is granted without fee provided that copies are not made or distributed for profit or commercial advantage and that copies bear this notice and the full citation on the first page. To copy otherwise, to republish, to post on servers or to redistribute to lists, requires prior specific permission and/or a fee.

SoICT 2012, August 23-24, 2012, Ha Long, Vietnam.

Copyright 2012 ACM 978-1-4503-1232-5/12/08 ...\$10.00.
Model predictive control (MPC) is an effective advanced control method since it can model multivariable systems and handle physical constraints in the control frame work. The principle of MPC is to solve a quadratic programming (QP) problem within the sampling period. This is a challenge when applying MPC to complex systems requiring fast response and/or small-scale devices where computational power and memory resources are limited. Recently, there have been research works on implementation of MPC using Application-Specific Integrated Circuit (ASIC) [5], and Field-Programmable Gate Array (FPGA) [11, 8, 3]. Although both of the two platforms can achieve high processing speeds, FPGAs are more flexible and have shorter design cycles than ASICs. The main drawbacks of FPGAs are their high power consumption and high cost. In addition to ASICs and FPGAs, MPC can be also implemented on general purpose microcontrollers $[7,6,1]$. Since a microcontrollers can be considered custom built mini computers in an IC, they are more flexible than ASICs and FPGAs in embedded system design. Other advantages of microcontrollers compared to FPGAs include their low power consumption and low cost. This paper proposes an implementation of MPC on low-power RISC microcontrollers, which can be widely applied to numerous miniaturized control systems.

Diabetes Mellitus is a widespread disease caused by high blood glucose in the patient's body (Diabetes.com). High blood sugar sets off processes that can lead to complications, like heart, kidney, and eye disease, or other serious problems. There are two types of Diabetes: Type 1 and Type 2 Diabetes. In addition to medication and/or insulin shots, Type 1 and some Type 2 patients are required to measure their blood sugar level everyday with the aid of a blood glucose monitor. A blood glucose monitor or glucose meter is a small portable battery-powered electronic device mainly used to determine the deviations of the patient's blood glucose level from the normal healthy level. There are many different types of blood glucose monitors in the market for diabetes management. Patients may be required to use lancets to draw blood from their finger. Recent models allow people to test the glucose level with blood extracted from other areas. The most recent models use sensor pod that is placed underneath the skin to constantly measure the blood sugar via an external monitoring device. Sensors from the latest model could be used continuously for up to a week. There are many interests in the healthcare industry to develop an implantable solution or artificial pancreas that could remove the patient's pains from blood extraction and needle insertion as well as better diabetes management out- 
comes. This paper also reports an application of our MPC controller for continuous diabetes management.

\section{MODEL PREDICTIVE CONTROL}

This section presents our extensions of the MPC design methodology from single-input and single-output (SISO) systems which is described in [15] to multi-input and multioutput (MIMO) systems.

MPC systems are designed based on a state-space model of the plant. Assuming the plant has $m$ inputs, $q$ outputs, and $n_{1}$ states, the formulation of the predictive control problem can be stated as below:

$$
\begin{aligned}
x_{m}(k+1) & =A_{m} x_{m}(k)+B_{m} u(k), \\
y(k) & =C_{m} x_{m}(k),
\end{aligned}
$$

where $u$ is the manipulated or input variable, $y$ is the process output, and $x_{m}$ is the state variable; $A_{m}, B_{m}$, and $C_{m}$ have dimensions of $n_{1} \times n_{1}, n_{1} \times m$, and $q \times n_{1}$, respectively.

Denoting $\Delta x_{m}(k)=x_{m}(k)-x_{m}(k-1), \Delta u(k)=u(k)-$ $u(k-1)$, and $x(k)=\left[\begin{array}{ll}\Delta x_{m}(k)^{T} & y(k)\end{array}\right]^{T}$, with a difference operation on both sides of (1a) and (1b), we obtain the following state-space model:

$$
\begin{aligned}
\overbrace{\left[\begin{array}{c}
\Delta x_{m}(k+1) \\
y(k+1)
\end{array}\right]}^{x(k+1)}=\overbrace{\left[\begin{array}{cc}
A_{m} & o_{m}^{T} \\
C_{m} A_{m} & I_{q \times q}
\end{array}\right]}^{A} \overbrace{\left[\begin{array}{c}
\Delta x_{m}(k) \\
y(k)
\end{array}\right]}^{x(k)} \\
+\underbrace{\left[\begin{array}{c}
B_{m} \\
C_{m} B_{m}
\end{array}\right]}_{B} \Delta u(k), \\
y(k)=\underbrace{\left[\begin{array}{ll}
o_{m} & I_{q \times q}
\end{array}\right]}_{C} \underbrace{\left[\begin{array}{c}
\Delta x_{m}(k) \\
y(k)
\end{array}\right]}_{x(k)},
\end{aligned}
$$

where $o_{m}$ is a $q \times n_{1}$ zero matrix, and $I_{q \times q}$ is the identity matrix with dimensions of $q \times q$.

\subsection{Solution of predictive control}

At the sampling instant $k_{i}$, we assume that the state variable $x\left(k_{i}\right)$ is available to provide the current information of the plant. Then, the future state variables are predicted for $N_{p}$ samples: $x\left(k_{i}+1 \mid k_{i}\right), x\left(k_{i}+2 \mid k_{i}\right), \ldots, x\left(k_{i}+N_{p} \mid k_{i}\right)$, and the optimal future control trajectory is described within $N_{c}$ samples $\left(N_{c} \leq N_{p}\right): \Delta u\left(k_{i}\right), \Delta u\left(k_{i}+1\right), \ldots, \Delta u\left(k_{i}+N_{c}-1\right)$. By defining

$$
\begin{gathered}
\Delta U=\left[\begin{array}{llll}
\Delta u\left(k_{i}\right)^{T} & \Delta u\left(k_{i}+1\right)^{T} & \cdots & \Delta u\left(k_{i}+N_{c}-1\right)^{T}
\end{array}\right]^{T} \\
Y=\left[\begin{array}{llll}
y\left(k_{i}+1 \mid k_{i}\right)^{T} & y\left(k_{i}+2 \mid k_{i}\right)^{T} & \cdots & y\left(k_{i}+N_{p} \mid k_{i}\right)^{T}
\end{array}\right]^{T},
\end{gathered}
$$

and sequentially calculating the predicted future state and output variables using the future control parameters, we obtain

$$
Y=F x\left(k_{i}\right)+\Phi \Delta U
$$

where

$$
F=\left[\begin{array}{llll}
C A & C A^{2} & \cdots & C A^{N_{p}}
\end{array}\right]^{T}
$$

and

$$
\Phi=\left[\begin{array}{cccc}
C B & 0 & \cdots & 0 \\
C A B & C B & \cdots & 0 \\
\vdots & \vdots & \cdots & \vdots \\
C A^{N_{p}-1} B & C A^{N_{p}-2} B & \cdots & C A^{N_{p}-N_{c}} B
\end{array}\right] .
$$

For a given set-point signal $r\left(k_{i}\right)=\left[r_{1}\left(k_{i}\right) r_{2}\left(k_{i}\right) \cdots r_{q}\left(k_{i}\right)\right]^{T}$ at sample time $k_{i}$, we need to find the optimal control parameter vector $\Delta U$ so that within the prediction horizon, the predicted output variables are as close as possible to the set-points. The cost function that reflects this control objective is defined as

$$
J=\left(R_{s}-Y\right)^{T}\left(R_{s}-Y\right)+\Delta U^{T} \bar{R} \Delta U,
$$

where $R_{s}=\left[\begin{array}{llll}I_{q \times q} & I_{q \times q} & \cdots & I_{q \times q}\end{array}\right]^{T} r\left(k_{i}\right)=\bar{R}_{s} r\left(k_{i}\right)$, and $\bar{R}$ is a block matrix with $m \times m$ blocks of $r_{\omega} I_{N_{c} \times N_{c}}\left(r_{\omega} \geq 0\right)$, in which $r_{\omega}$ is a tuning parameter, and $I_{N_{c} \times N_{c}}$ is the $N_{c} \times N_{c}$ identity matrix.

From (4) and (7), by solving $\frac{\partial J}{\partial \Delta U}=0$ for the minimum of $J$, the optimal solution is:

$$
\Delta U=\left(\Phi^{T} \Phi+\bar{R}\right)^{-1} \Phi^{T}\left(R_{s}-F x\left(k_{i}\right)\right) .
$$

Note that only the first $m$ elements of $\Delta U$, i.e. $\Delta u\left(k_{i}\right)$, will be utilized. At next sample time, the new state variable $x\left(k_{i}+1\right)$ is measured and used to calculate the new control trajectory.

\subsection{State estimate predictive control}

At time $k_{i}$, the state variable $x\left(k_{i}\right)$ is assumed to be available. However, in practice, not all state variables are available or measurable. A solution to this issue is to estimate the state variable $x(k)$ using a state observer. We calculate the state variable $\hat{x}_{m}(k)$ based on the plant model in (1), and use the error signal of output as a feedback to improve the estimation as below

$$
\hat{x}_{m}(k+1)=\underbrace{A_{m} \hat{x}_{m}(k)+B_{m} u(k)}_{\text {model }}+\underbrace{K_{o b}\left(y(k)-C_{m} \hat{x}_{m}(k)\right)}_{\text {correctionterm }},
$$

where $K_{o b}$ is the observer gain matrix.

By substituting (1b) into (9), we deduce that the error $\tilde{x}_{m}(k)=x_{m}(k)-\hat{x}_{m}(k)$ satisfies the difference equation:

$$
\begin{aligned}
\tilde{x}_{m}(k+1) & =A_{m} \tilde{x}_{m}(k)-K_{o b} C_{m} \tilde{x}_{m}(k) \\
& =\left(A_{m}-K_{o b} C_{m}\right) \tilde{x}_{m}(k) .
\end{aligned}
$$

For a given initial error state $\tilde{x}_{m}(0) \neq 0$, we have

$$
\tilde{x}_{m}(k)=\left(A_{m}-K_{o b} C_{m}\right)^{k} \tilde{x}_{m}(0) .
$$

If the observer gain $K_{o b}$ is chosen so that the error system matrix $A_{m}-K_{o b} C_{m}$ has all eigenvalues inside the unit circle, then the error system (11) is stable and $\left\|\tilde{x}_{m}(k)\right\| \rightarrow 0$ as $k \rightarrow \infty$. This means that the estimated state variable $\hat{x}_{m}(k)$ converges to $x_{m}(k)$.

At sample time $k_{i}$, with the information of $\hat{x}\left(k_{i}\right)$ replacing $x\left(k_{i}\right)$, the estimation of state variable in (2) is modified as

$$
\hat{x}\left(k_{i}+1\right)=A \hat{x}\left(k_{i}\right)+B \Delta u\left(k_{i}\right)+K_{o b}\left(y\left(k_{i}\right)-C \hat{x}\left(k_{i}\right)\right) .
$$

The cost function and optimal solution are similar to that of (7) and (8), respectively, except that the term $x(\cdot)$ is replaced by $\hat{x}(\cdot)$. 


\subsection{MPC with constraints}

We need to modify the above solution if operational constraints are introduced to the model. Typical constraints are:

(1) Constraints on rate of change of all the control variables within the future control trajectory started from timestep $k_{i}$ :

$$
\Delta U^{\min } \leq \Delta U \leq \Delta U^{\max },
$$

where $\Delta U$ was defined in (3a); $\Delta U^{\text {min }}$ and $\Delta U^{\max }$ are column vectors with $N_{c}$ elements of the lower limits $\Delta u^{\min }=\left[\begin{array}{llll}\Delta u_{1}^{\min } & \Delta u_{2}^{\min } & \cdots & \Delta u_{m}^{\min }\end{array}\right]$ and the upper limits $\Delta u^{\max }=\left[\begin{array}{llll}\Delta u_{1}^{\max } & \Delta u_{2}^{\max } & \cdots & \Delta u_{m}^{\max }\end{array}\right]$, respectively.

(2) Constraints on amplitude of all the control variables within the future control trajectory:

$$
U^{\min } \leq U \leq U^{\max },
$$

where $U=\left[\begin{array}{llll}u\left(k_{i}\right)^{T} & u\left(k_{i}+1\right)^{T} & \cdots & u\left(k_{i}+N_{c}-1\right)^{T}\end{array}\right]^{T}$; $U^{\text {min }}$ and $U^{\max }$ are column vectors with $N_{c}$ elements of the

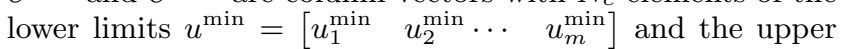
limits $u^{\max }=\left[\begin{array}{llll}u_{1}^{\max } & u_{2}^{\max } & \cdots & u_{m}^{\max }\end{array}\right]$, respectively.

(3) Constraints on the outputs within the prediction trajectory:

$$
Y^{\min } \leq Y \leq Y^{\max },
$$

where $Y$ was defined in (3b); $Y^{\text {min }}$ and $Y^{\max }$ are column vectors with $N_{p}$ elements of the lower limits $y^{\min }=\left[\begin{array}{llll}y_{1}^{\min } & y_{2}^{\min } & \cdots & y_{q}^{\min }\end{array}\right]$ and the upper limits $y^{\max }=\left[\begin{array}{llll}y_{1}^{\max } & y_{2}^{\max } & \cdots & y_{q}^{\max }\end{array}\right]$, respectively.

The constraints in (13) can be rewritten in a matrix form:

$$
\left[\begin{array}{c}
-I \\
I
\end{array}\right] \Delta U \leq\left[\begin{array}{c}
-\Delta U^{\min } \\
\Delta U^{\max }
\end{array}\right]
$$

In (14), $U=\left[\begin{array}{llll}u\left(k_{i}\right)^{T} & u\left(k_{i}+1\right)^{T} & \cdots & u\left(k_{i}+N_{c}-1\right)^{T}\end{array}\right]^{T}$, which can be expressed as

$$
U=\underbrace{\left[\begin{array}{c}
I \\
I \\
\vdots \\
I
\end{array}\right]}_{C_{1}} u\left(k_{i}-1\right)+\underbrace{\left[\begin{array}{cccc}
I & O & \cdots & O \\
I & I & \cdots & O \\
\vdots & \vdots & \ddots & \vdots \\
I & I & \cdots & I
\end{array}\right]}_{C_{2}} \underbrace{\left[\begin{array}{c}
\Delta u\left(k_{i}\right) \\
\Delta u\left(k_{i}+1\right) \\
\vdots \\
\Delta u\left(k_{i}+N_{c}-1\right)
\end{array}\right]}_{\Delta U},
$$

where $I$ and $O$ are the identity and zero matrices with dimension of $m \times m$. From (17), we can represent (14) as

$$
\left[\begin{array}{c}
-C_{2} \\
C_{2}
\end{array}\right] \Delta U \leq\left[\begin{array}{c}
-U^{\min }+C_{1} u\left(k_{i}-1\right) \\
U^{\max }-C_{1} u\left(k_{i}-1\right)
\end{array}\right] .
$$

From (4), the output constraints can be rewritten as

$$
\left[\begin{array}{c}
-\Phi \\
\Phi
\end{array}\right] \Delta U \leq\left[\begin{array}{c}
-Y^{\min }+F x\left(k_{i}\right) \\
Y^{\max }-F x\left(k_{i}\right)
\end{array}\right]
$$

Finally, solving the MPC with constraints is to find $\Delta U$ that minimizes the cost function in (7) subject to the inequality constraints in (16), (18), and (19). This is a quadratic programming problem with linear inequality constraints:

$$
\begin{aligned}
& J=\frac{1}{2} x^{T} E x+x^{T} F, \\
& M x \leq \gamma,
\end{aligned}
$$

where $E$ and $F$ can be inferred from (7); $M$ and $\gamma$ are a matrix and a vector reflecting the constraints. In the case that the constraints are fully imposed, $M$ and $\gamma$ have $4 m N_{c}+$ $2 q N_{p}$ rows.

The optimization problem in (20) can be solved using various methods. This work uses a primal-dual interior-point algorithm [13] employing the predictor-corrector method [16] which is computational and resource efficient.

\section{LINEAR INEQUALITY CONSTRAINED QUADRATIC PROGRAMMING}

This section describes a primal-dual interior-point method to solve the quadratic program shown in (20) where $x$ is a $n \times 1$ vector (in our problem, $x$ is $\Delta U$, and $n=m \times N_{c}$ ), $E$ is a $n \times n$ matrix, $F$ is a $n \times 1$ vector, $M$ is a $l \times n$ matrix, where $l$ is the number of inequality constraints, and $\gamma$ is a $l \times 1$ vector.

Denoting $\lambda$ as the Lagrange multipliers vector of the optimization problem, and introducing the slack vector $s=$ $\gamma-M x, s \geq 0$, then the Karush-Kuhn-Tucker (KKT) conditions for this problem can be represented as

$$
\begin{array}{r}
\Psi(x, \lambda, s)=\left[\begin{array}{c}
E x+F+M^{T} \lambda \\
s+M x-\gamma \\
\Lambda S e
\end{array}\right]=0, \\
\lambda, s \geq 0,
\end{array}
$$

where $\Lambda=\operatorname{diag}\left(\lambda_{1}, \lambda_{1}, \ldots, \lambda_{l}\right), S=\operatorname{diag}\left(s_{1}, s_{2}, \ldots, s_{l}\right)$, and $e=[1,1, \ldots, 1]^{T}$.

In order to find the solutions $\left(x^{*}, \lambda^{*}, s^{*}\right)$, primal-dual interiorpoint methods apply variants of Newton's method to (21a) and modify the search directions and step lengths so that the inequalities (21b) satisfied strictly, i.e., $\lambda>0$ and $s>0$, at each iteration. From the current point $(x, \lambda, s)$, we can obtain the search direction $(\Delta x, \Delta \lambda, \Delta s)$ by solving

$$
\Psi^{\prime}(x, \lambda, s)\left[\begin{array}{lll}
\Delta x & \Delta \lambda & \Delta s
\end{array}\right]^{T}=-\Psi(x, \lambda, s),
$$

where $\Psi^{\prime}=\left[\begin{array}{ccc}E & M^{T} & 0 \\ M & 0 & I \\ 0 & S & \Lambda\end{array}\right]$ is the Jacobian of $\Psi$. After $(\Delta x, \Delta \lambda, \Delta s)$ is obtained, the new iterate is calculated as

$$
(x, \lambda, s)+\alpha(\Delta x, \Delta \lambda, \Delta s),
$$

where the step length $\alpha \in[0,1]$ is determined so that the condition $(21 \mathrm{~b})$ is hold.

However, in practice, if following the pure Newton direction (also known as the affine-scaling direction) in (22), we only can obtain a small step length, i.e., $\alpha<<1$, before the condition $(\lambda, s)>0$ is violated. Hence, the convergence speed is rather slow. To overcome this issue, primal-dual interior-point methods bias the Newton direction toward the interior of the orthant $(\lambda, s) \geq 0$ and keep the components of $(\lambda, s)$ away from the boundary of the orthant. Typically, the search direction is aimed to a point whose pairwise products $\lambda_{i} s_{i}$ are reduced to a lower average value. The duality measure $\mu=\frac{\lambda^{T} s}{l}$ is used to estimate the reduction speed of $\lambda^{T} s$. The Newton search direction is then modified toward a point for which $\lambda_{i} s_{i}=\sigma \mu$, where $\mu$ is the current duality measure and $\sigma \in[0,1]$ is termed a centering parameter.

The values of $\sigma$ and $\alpha$ are two important factors to the performance of the method. Different strategies of choosing these parameters lead to a wide range of primal-dual interior-point methods. Our method is based on the predictorcorrector method proposed by Mehrotra [13], which was proven 
to be efficient in practice. Details of the algorithm are presented in Algorithm 1.

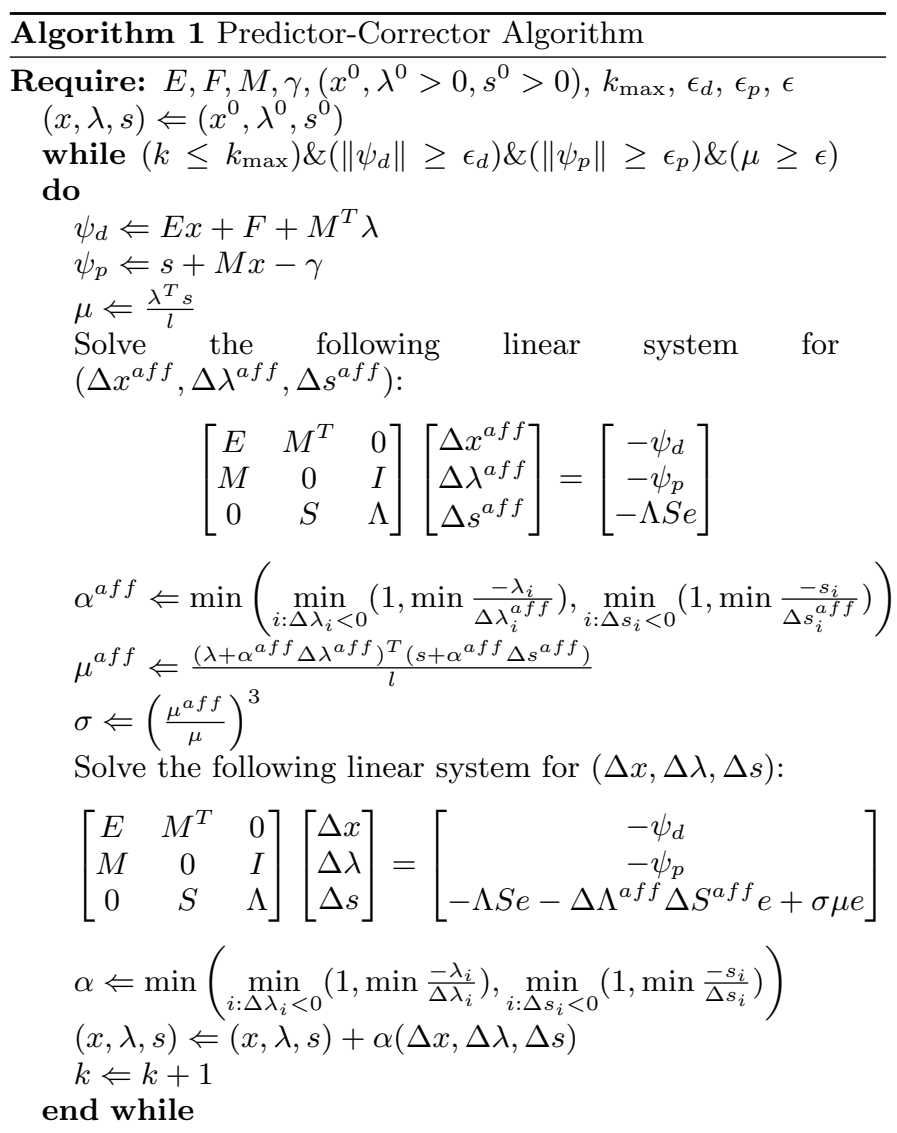

It should be noted that the two linear systems in Algorithm 1 have a same coefficient matrix. Thus, the matrix factorization needs to be computed only once, leading to a relatively low cost of solving the second system.

\section{MODIFIED MINIMAL MODEL}

There are many mathematical blood glucose models proposed in the literature [2]. Among them, the minimal model proposed by Bergman et al. (1981) [4] is the most popular model due to its simplicity and physiological accuracy. The blood glucose model in this work is based on the Fisher model [9], which is a modified version of the minimal model.

In addition to changes on the third equation of the minimal model made by Fisher (1991) in order to adapt the model to type I diabetic, that is removing the insulin secretion and adding an insulin infusion term, we add one more equation which is adapted from [12] to represent a relationship between plasma glucose concentration and subcutaneous glucose. This is due to in continuous glucose monitoring in practice, most glucose measurements are obtained via the subcutaneous layer.
The model equations used in our work are

$$
\begin{aligned}
\frac{d G}{d t} & =-p_{1}\left(G(t)-G_{b}\right)-X(t) G(t)+P(t), \\
\frac{d X}{d t} & =-p_{2} X(t)+p_{3}\left(I(t)-I_{b}\right), \\
\frac{d I}{d t} & =-n I(t)+U(t) / V_{I}, \\
\frac{d G_{s c}}{d t} & =\frac{G(t)-G_{s c}(t)}{5}-R_{u t l n},
\end{aligned}
$$

where the model parameters are described in Table 4.

The following discrete model is obtained by linearizing the model with the steady-state values of $\left(G, X, I, G_{s c}\right)=$ $\left(G_{b}, 0, I_{b}, G_{b s c}\right)$ :

$$
\begin{aligned}
x_{k+1} & =A x_{k}+B u_{k}+D d_{k}, \\
y_{k} & =C x_{k},
\end{aligned}
$$

where $x, u$ and $y$ represent the state variable, input and output of the system, respectively; $k$ is the discrete timestep.

With the model parameters adopted from [12]:

$$
\begin{array}{lll}
p_{1}=0.028735 & p_{2}=0.028344 & p_{3}=5.035 \times 10^{-5} \\
G_{b}=81.3 & G_{b s c}=77.6 & I_{b}=15 \\
V_{I}=12 & n=5 / 54 &
\end{array}
$$

and the sampling time of 5 minutes, the parameter matrices in $(25)$ are:

$$
\begin{aligned}
A & =\left[\begin{array}{cccc}
0.8662 & -352.4422 & -0.0400 & 0 \\
0 & 0.8679 & 0.0002 & 0 \\
0 & 0 & 0.6294 & 0 \\
0.5819 & -135.0405 & -0.0111 & 0.3679
\end{array}\right], \\
B & =\left[\begin{array}{c}
-0.0059 \\
0.0000 \\
0.3335 \\
-0.0013
\end{array}\right], C=\left[\begin{array}{llll}
0 & 0 & 0 & 1
\end{array}\right], D=\left[\begin{array}{llll}
5 & 0 & 0 & 0
\end{array}\right]^{T} .
\end{aligned}
$$

The term $P(t)$ in (24) represents the rate of glucose entering the blood from intestinal absorption after a meal, and can be estimated based on the following meal model [10]:

$$
U_{G}(t)=\frac{D_{G} A_{G} t e^{-t / t_{\max , G}}}{t_{\max , G}^{2}},
$$

where $D_{G}(m g)$ is the amount of carbohydrates digested, $A_{G}$ represents the carbohydrate bioavailability, and $t_{\max , G}$ ( $\min )$ is the time from the beginning of the meal consumption until the absorption rate reaches its maximum. $A_{G}$ and $t_{\max , G}$ are chosen as 0.8 and 40 minutes, respectively, as in [10]. Hence, the term $d_{k}$ in (25) is determined as

$$
d_{k}=\left\{\begin{array}{cc}
0.00002 D_{G}\left(k-k_{G}\right) e^{-0.125\left(k-k_{G}\right)} & \text { if } k \geq k_{G}, \\
0 & \text { otherwise }
\end{array}\right.
$$

where $k_{G}$ is the time-step at which the meal disturbance is implemented.

The set-point is chosen as $G_{b s c}(77.6 \mathrm{mg} / \mathrm{dL})$ in our experiments. In addition, due to limitations on the range and possible changes of insulin infusion rate that can be enforced by a common insulin pump, and conditions of the output to avoid hypoglycemia and extreme hyperglycemia, the follow- 
Table 1: Model Parameters

\begin{tabular}{|l|l|l|}
\hline Parameter & Unit & Description \\
\hline$G(t)$ & $m g / d L$ & Plasma glucose concentration \\
$G_{s c}(t)$ & $m g / d L$ & Subcutaneous glucose concentration \\
$X(t)$ & $m i n^{-1}$ & Insulin variable for the remote compartment \\
$I(t)$ & $m U / L$ & Plasma insulin concentration \\
$G_{b}$ & $m g / d L$ & Basal value of plasma glucose concentration \\
$G_{b s c}$ & $m g / d L$ & Basal value of subcutaneous glucose concentration \\
$I_{b}$ & $m U / L$ & Basal value of plasma insulin concentration \\
$P(t)$ & $(m g / d L) m_{i n}^{-1}$ & Climbing rate of blood glucose due to a meal disturbance \\
$U(t)$ & $m U / m i n$ & Exogenous insulin infusion rate \\
$V_{I}$ & $L$ & Insulin distribution volume \\
$p_{1}$ & $m i n^{-1}$ & Insulin-independent constant rate of glucose uptake \\
$p_{2}$ & $\min ^{-1}$ & Rate of clearance of active insulin \\
$p_{3}$ & $L /\left(\mathrm{min}^{2} m U\right)$ & Increase in uptake ability caused by insulin \\
$R_{u t l n}$ & $(m g / d L) \mathrm{min}^{-1}$ & Tissue rate of utilization \\
\hline
\end{tabular}

ing constraints are also imposed:

$$
\begin{array}{r}
0 \leq u \leq 80 \mathrm{mU} / \mathrm{min}, \\
-16.7 \leq \Delta u \leq 16.7 \mathrm{mU} / \mathrm{min}, \\
60 \leq y \leq 180 \mathrm{mg} / \mathrm{dL} .
\end{array}
$$

Since measurements of $X$ are physically unavailable, and those of $I$ are inaccessible in practice, we need to estimate the state variable $x$ using an observer. Hence, the optimization problem is listed in (20), where all the parameter matrices can be easily inferred from (7), (12), (25), (27), and (28).

\section{MPC-ON-A-CHIP IMPLEMENTATION}

We implemented the constrained MPC algorithm on a Texas Instruments (TI) MSP430FG4618 microcontroller. Thi is a ultralow-power 16-bit RISC-architecture microcontroller consisting of $116 \mathrm{~KB}+256 \mathrm{~B}$ Flash ROM, $8 \mathrm{~KB}$ RAM, and five low-power modes, which is optimized to achieve extended battery life in portable measurement applications. It should be noted that our implementation can be applied to any generic RISC microcontroller. In addition, it is easily to port our code to other TI series or other platforms since it was developed using the standard $\mathrm{C}$ language.

Main functions. As seen from Sections 2 and 3, most of the calculations are based on matrix operations and solving systems of linear equations. We implemented these calculations as functions which are described in Table 5 .

Dynamic memory-allocation. Since the dimensions of the matrices vary based on a specific application, the functions in Table 5 should use dynamic memory-allocation to store matrices and intermediate values. However, due to limited memory of microcontrollers, "dynamic heap memory allocation shall not be used" as indicated in [14] (rule 118). Hence, we should not use the built-in memory-allocation routines in $\mathrm{C}$, e.g, malloc, realloc, calloc or free. Instead, we propose the use of a global array as a buffer shared between the main function and all other sub-routines to store dynamic variables and intermediate values. For any function which requires a dynamic memory-allocation, it will be provided by the caller function a part of the global buffer through an input pointer. The caller function needs to determine the address of the buffer allocated to the called function. The size of the global array is set based on the imple-
Listing 1: Dynamic Memory-Allocation Illustration

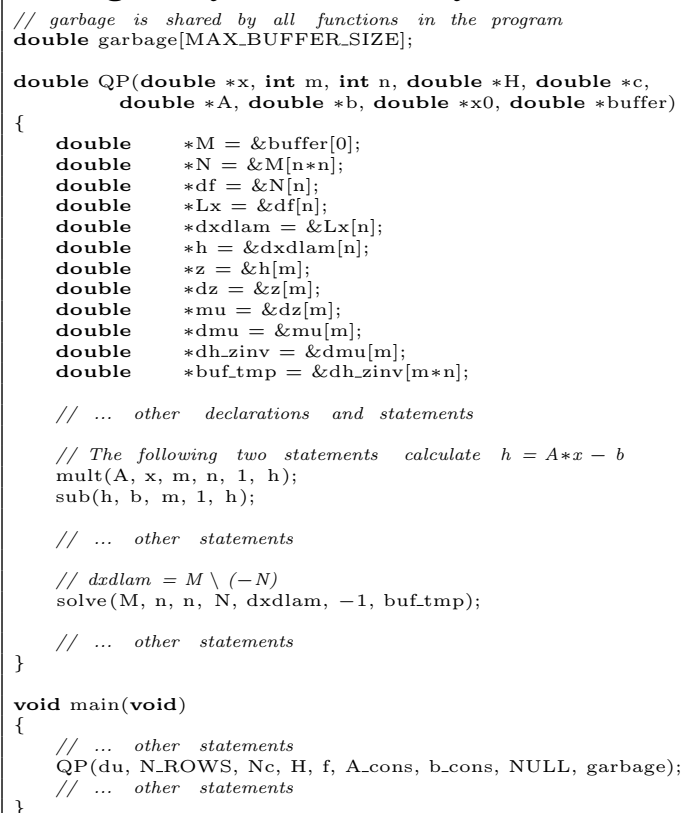

mentation of all the functions and the size of the physical SRAM memory of the microcontroller.

Listing 1 illustrates our dynamic memory-allocation mechanism. As in Table 5, solve and QP are two functions requiring buffers to store intermediate values. Dynamic variables in QP are allocated through the input address buffer and the size parameters $m$ and $n$ as from lines 7 to 18 in the listing. When solve is called by QP, a buffer is provided to solve through the array buf_tmp which has already been allocated in QP (line 29). When QP is called by the main function, its buffer is assigned as the global buffer garbage (line 37).

\section{RESULTS AND DISCUSSION}

A Virtual Patient using the modified minimal model was implemented on a computer to verify the accuracy of the embedded MPC algorithm. The Virtual Patient communicates with the MPC microcontroller through serial communica- 
Table 2: Main Functions

\begin{tabular}{|c|c|c|}
\hline Function prototype & Description & Parameters \\
\hline void zeros $\left(\right.$ double ${ }^{*} \mathrm{~A}$, int $\left.\mathrm{n}\right)$ & $\begin{array}{l}\text { Set a square matrix to a zero ma- } \\
\text { trix }\end{array}$ & $\begin{array}{l}\text { A: [out] an } n \times n \text { matrix to be set, } \mathrm{n} \text { : [in] size of } \\
\text { A }\end{array}$ \\
\hline void eye $\left(\right.$ double ${ }^{*} \mathrm{~A}$, int $\left.\mathrm{n}\right)$ & $\begin{array}{l}\text { Set a square matrix to identity } \\
\text { matrix }\end{array}$ & $\begin{array}{l}\text { A: [out] an } n \times n \text { matrix to be set, } \mathrm{n} \text { : [in] size of } \\
\text { A }\end{array}$ \\
\hline $\begin{array}{l}\text { void copy(double }{ }^{*} \text { src, } \text { double } \\
*_{\text {dst, int size); }}\end{array}$ & $\begin{array}{l}\text { Copy elements between two matri- } \\
\text { ces }\end{array}$ & $\begin{array}{l}\text { src: [in] matrix to copy from, dst: [out] destina- } \\
\text { tion matrix, size: [in] number of elements to copy }\end{array}$ \\
\hline $\begin{array}{l}\text { void transpose }\left(\text { double }{ }^{*} \text { src, int }\right. \\
\left.m \text {, int } n \text {, double }{ }^{*} \text { dst }\right) ;\end{array}$ & Compute matrix transpose & $\begin{array}{l}\text { src: [in] an } m \times n \text { input matrix, dst: [out] the } \\
n \times m \text { output matrix }\end{array}$ \\
\hline 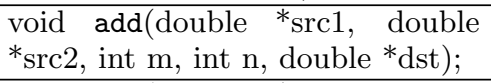 & Add two matrices & $\begin{array}{l}\text { src1, src2: [in] two input matrices, dst: [out] the } \\
\text { sum matrix, } m \times n \text { : size of the three matrices }\end{array}$ \\
\hline 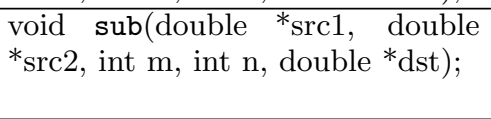 & Subtract two matrices & $\begin{array}{l}\text { src1: [in] minuend matrix, src2: [in] subtrahend } \\
\text { matrix, dst: [out] the difference matrix, } m \times n \text { : } \\
\text { size of the three matrices }\end{array}$ \\
\hline $\begin{array}{l}\text { void mult(double }{ }^{*} \text { src1, double } \\
{ }^{*} \text { src } 2 \text {, int } \mathrm{m} \text {, int } \mathrm{n} \text {, int } \mathrm{p} \text {, double } \\
{ }^{*} \text { dst); }\end{array}$ & Multiply two matrices & $\begin{array}{l}\text { src1: [in] an } m \times n \text { input matrix, src2: [in] an } n \times p \\
\text { input matrix, dst: [out] the } m \times p \text { product matrix }\end{array}$ \\
\hline $\begin{array}{l}\text { void solve(double }{ }^{*} \mathrm{~A} \text {, int } \mathrm{m} \text {, int } \\
\mathrm{n} \text {, double }{ }^{*} \text { b, double }{ }^{*} \mathrm{x} \text {, double } \\
\text { thresh, double *buffer }) ;\end{array}$ & $\begin{array}{l}\text { Solve a system of linear equations: } \\
A x=b\end{array}$ & $\begin{array}{l}\text { A: [in] an } m \times n \text { matrix, b: [in] a } m \times 1 \text { vector, } \\
\text { x: [out] the } n \times 1 \text { solution vector, thresh: [in] a } \\
\text { threshold for checking singular values, buffer: [in] } \\
\text { an array (of } m \times(n+1)+n \times(n+2) \text { elements) } \\
\text { to store intermediate values }\end{array}$ \\
\hline $\begin{array}{l}\text { double } \mathrm{QP}\left(\text { double }{ }^{*} \mathrm{x}, \text { int } \mathrm{m} \text {, int }\right. \\
\mathrm{n} \text {, double }{ }^{*} \mathrm{H}, \text { double }{ }^{*} \mathrm{c} \text {, double } \\
{ }^{*} \text { A, double }{ }^{*} \mathrm{~b} \text {, double }{ }^{*} \mathrm{x} 0 \text {, double } \\
\left.{ }^{*} \text { buffer }\right)\end{array}$ & $\begin{array}{l}\text { Solve a quadratic programming } \\
\text { problem as minimizing } \\
J=\frac{1}{2} x^{T} H x+x^{T} c \\
\text { subject to } A x \leq b\end{array}$ & $\begin{array}{l}\text { x: [out] the } n \times 1 \text { solution vector, H: [in] an } n \times n \\
\text { matrix, c: [in] an } n \times 1 \text { vector, A: [in] an } m \times n \\
\text { matrix, b: [in] an } m \times 1 \text { vector, x0: [in] an initial } \\
\text { solution, buffer: [in] an array to store intermediate } \\
\text { values }\end{array}$ \\
\hline
\end{tabular}
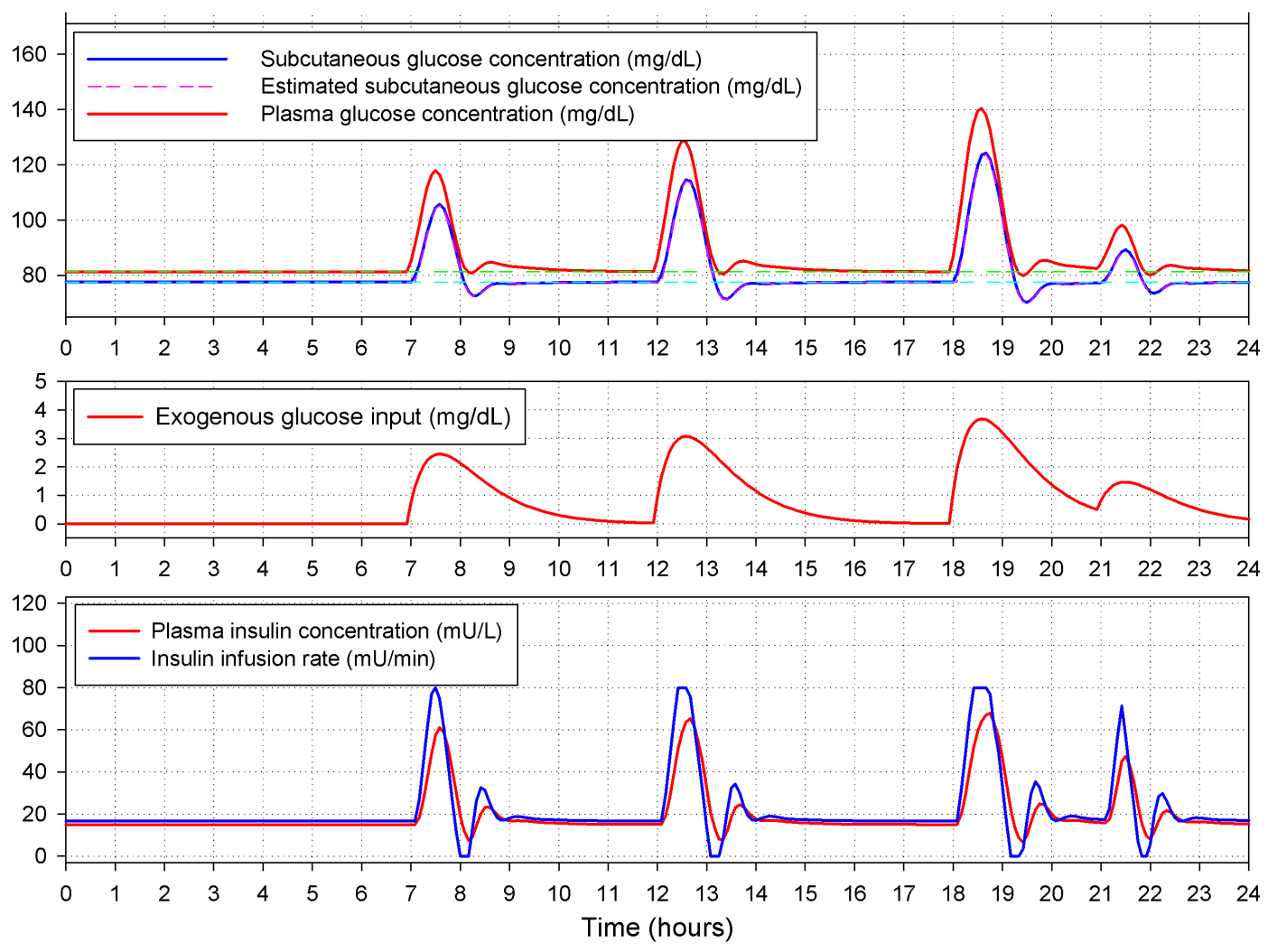

Figure 1: Simulation Results. 
tions. Figure 1 shows the simulation results for a patient who has breakfast, lunch, dinner, and supper at 7:00, 12:00, 18:00, and 21:00 respectively. The corresponding meal sizes are $40 \mathrm{~g}, 50 \mathrm{~g}, 60 \mathrm{~g}$, and $20 \mathrm{~g}$ of carbohydrates, respectively. As seen in Figure 1, the patient blood glucose reaches the maximum level after the meal starts about 30-40 minutes. However, with a prediction window of 300 minutes $\left(N_{P}=60\right)$ and appropriate meal size, the MPC-based artificial pancreas is able to bring down the glucose level to the basal value $3-3.5$ hours after the meal.

The binary code of the MPC and serial communications on the MSP430F5438A microcontroller is $24 \mathrm{~KB}$, which is smaller than that of other implementations $[7,6]$. The time for computation and communications during each time-step was less than 1 second on average. With longer prediction and control windows $\left(N_{P}=80, N_{C}=4\right)$, the computation speed of our system is comparable with that of the other designs $[7,6,1]$. Other advantages of our implementation compared to these designs are that (1) all typical operational constraints, and (2) the estimation of unmeasurable states are supported. In addition, this system is highly energy efficient since most of the time the processor is on standby mode with the power consumption rate of $2.1 \mu \mathrm{A}$ at $3.0 \mathrm{~V}$. The power consumption rate in active mode is about $230 \mu \mathrm{A} / \mathrm{MHz}$ at $8 \mathrm{MHz}, 3.0 \mathrm{~V}$. The microcontroller takes less than $5 \mu$ s to be active from standby mode.

\section{CONCLUSION}

We have proposed an implementation of MPC with constraints on a microcontroller, and applied this implementation on the development of a real-time implantable closeloop glucose control system. The experimental results show that our embedded MPC microcontroller has low power consumption and is capable of performing the computational efficient MPC algorithm accurately and in real-time.

\section{REFERENCES}

[1] A. K. Abbes, F. Bouani, and M. Ksouri. A microcontroller implementation of constrained model predictive control. I. J. Electr. Electron. Eng., 5(3):199-206, 2006.

[2] N. P. Balakrishnan, G. P. Rangaiah, and L. Samavedham. Review and analysis of blood glucose (BG) models for type 1 diabetic patients. Ind. Eng. Chem. Res, 50(21):12041-12066, Sep 2011.

[3] K. Basterretxea and K. Benkrid. Embedded high-speed model predictive controller on a FPGA. In Proc. AHS 2011, pages 327-335, Jun 2011.

[4] R. N. Bergman, L. S. Phillips, and C. Cobelli. Physiologic evaluation of factors controlling glucose tolerance in man: measurement of insulin sensitivity and beta-cell glucose sensitivity from the response to intravenous glucose. J. Clin. Invest, 68(6):1456-1467, Dec 1981.

[5] L. G. Bleris, J. Garcia, M. V. Kothare, and M. G. Arnold. Towards embedded model predictive control for System-on-a-Chip applications. J. Process. Contr., 16(3):255-264, Mar 2006.

[6] L. G. Bleris and M. V. Kothare. Implementation of model predictive control for glucose regulation on a general purpose microprocessor. In Proc. CDC-ECC 2005, pages 5162-5167, Dec 2005.
[7] L. G. Bleris and M. V. Kothare. Real-time implementation of model predictive control. In Proc. $A C C$ 2005, volume 6, pages 4166-4171, Aug 2005.

[8] L. G. Bleris, P. D. Vouzis, M. G. Arnold, and M. V. Kothare. A co-processor FPGA platform for the implementation of real-time model predictive control. In Proc. ACC 2006, pages 1912-1917, Jun 2006.

[9] M. E. Fisher. A semiclosed-loop algorithm for the control of blood glucose levels in diabetics. IEEE Trans. Biomed. Eng, 38(1):57-61, Jan 1991.

[10] R. Hovorka, V. Canonico, L. J. Chassin, U. Haueter, W. Massi-Benedetti, M. O. Federici, T. R. Pieber, H. C. Schaller, L. Schaupp, T. Vering, and M. E. Wilinska. Nonlinear model predictive control of glucose concentration in subjects with type 1 diabetes. Physiol. Meas., 25(4):905-920, Aug 2004.

[11] K. V. Ling, S. P. Yue, and J. M. Maciejowski. A FPGA implementation of model predictive control. In Proc. ACC 2006, pages 1930-1935, Jun 2006.

[12] S. M. Lynch and B. W. Bequette. Model predictive control of blood glucose in type I diabetics using subcutaneous glucose measurements. In Proc. ACC 2002, pages 4039-4043, May 2002.

[13] S. Mehrotra. On the implementation of a primal-dual interior point method. SIAM J. Optim., 2(4):575-601, 1992.

[14] MISRA. Guidelines for the use of the $C$ language in critical systems. MIRA Ltd., Oct 2004.

[15] L. Wang. Model predictive control system design and implementation using MATLAB. Advances in Industrial Control. Springer-Verlag London, 2009.

[16] S. J. Wright. Primal-dual interior-point methods. SIAM, 1997. 